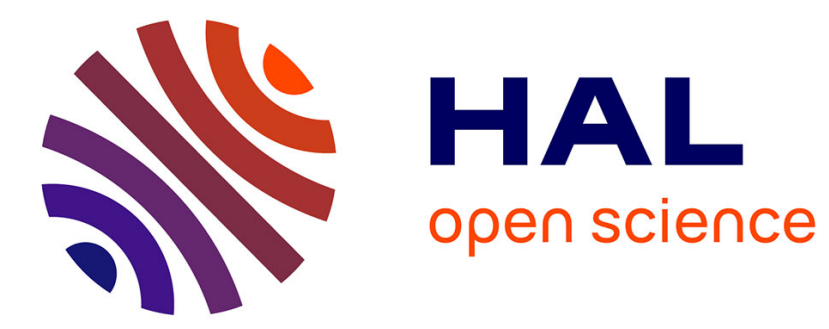

\title{
UNE NOUVELLE VISION DES BRUITS DE CIRCULATION AVEC LES P.A.I.S.
}

\author{
M. Maurin, L. Messiaen
}

\section{To cite this version:}

M. Maurin, L. Messiaen. UNE NOUVELLE VISION DES BRUITS DE CIRCULATION AVEC LES P.A.I.S.. Journal de Physique IV Proceedings, 1992, 02 (C1), pp.C1-463-C1-466. 10.1051/jp4:1992199 . jpa-00251271

\section{HAL Id: jpa-00251271 https://hal.science/jpa-00251271}

Submitted on 1 Jan 1992

HAL is a multi-disciplinary open access archive for the deposit and dissemination of scientific research documents, whether they are published or not. The documents may come from teaching and research institutions in France or abroad, or from public or private research centers.
L'archive ouverte pluridisciplinaire HAL, est destinée au dépôt et à la diffusion de documents scientifiques de niveau recherche, publiés ou non, émanant des établissements d'enseignement et de recherche français ou étrangers, des laboratoires publics ou privés. 


\title{
UNE NOUVELLE VISION DES BRUITS DE CIRCULATION AVEC LES P.A.I.S.
}

\author{
M. MAURIN et L. MESSIAEN* \\ Institut National de Recherche sur les Transports et leur Sécurité, Laboratoire Energie Nuisances, \\ INRETS-LEN, Case 24, F-69675 Bron cedex, France \\ *ECL, Laboratoire d'Acoustique, BP. 163, F-69131 Ecully Cedex, France
}

\begin{abstract}
RESUME - Nous explorons les ressources d'une nouvelle modélisation des niveaux de bruit routier, les mesures à proximité des voies routières chargées montrent un accord satisfaisant.

ABSTRACT - We investigate a new road noise modelling. It concerns the acoustic power $V_{t}=\left(p(t) / p_{0}\right)^{2}$ and its integral $X_{t}=\int V_{t} d t$. Because Leq definition $10 \log (\Delta X / \Delta t)$ it is convenient to introduce processes with independent and stationnary increments PISI. Their general form is known (Lévy, Khintchine), after some properties we develop estimators for $\Delta \mathrm{X}$ law with some special statistics of measured Leq. $q_{i}$. Road noise measures are made in several urban localisations, near by highways and without any reflexions (say when independence between successive increments of $\int_{t_{t}} d t$ may reasonnably be postulated) one gets estimators in rather good agreement with PISI models.
\end{abstract}

Parmi les bruits de l'Environnement les niveaux de bruit de la circulation routière présentent une certaine stabilité pendant les heures ouvrables de la journée ; pour chaque site et sur une plage temporelle comprise environ entre 7 et $8 \mathrm{~h}$ le matin, 19 et $20 \mathrm{~h}$ le soir, ils oscillent sans trop d'irrégularités autour d'un palier moyen [1]. Par ailleurs il est classique de les mesurer par des niveaux de bruit équivalents Leq, soit sur une longue durée, soit sur une ou plusieurs courtes durées $[2,3,4]$.

\section{I - LES PAIS ET LEURS PROPRIETES.}

I.1 - Compte tenu de la définition du Leq : $\operatorname{Leq}_{\Delta t}=10 \log \left\{\frac{1}{\Delta t} \int_{t}^{t+\Delta t} V_{u} d u\right\}$ avec $V_{t}=10^{L(t) / 10}=\left(p(t) / p_{0}\right)^{2}$, $L(t)$ le niveau de bruit instantané, $p(t)$ la pression acoustique, $p_{0}$ la pression de référence $210^{-5} \mathrm{~Pa}$ et $V_{1}$ la "puissance" acoustique [5], mais aussi des sonomètres intégrateurs actuels qui mesurent et stockent les niveaux équivalents Leq sur toutes durées successives, il semble pertinent de s'occuper des accroissements de l'intégrale de puissance $\Delta\left(\int \mathrm{V}_{\mathrm{t}} \mathrm{dt}\right)$ sur toute durée $\Delta \mathrm{t}$. C'est précisément le cas quand on modélise le signal intégral $X_{t}=\int V_{t} d t$ avec tout processus défini en terme de ses accroissements ; parmi ceux-ci figurent les Processus à Accroissements Indépendants et Stationnaires (PAIS) qui ont été l'une des causes de l'étude systématique des processus, et dont leurs principales propriétés ont été exposées par Lévy puis par Khintchine [Ø]. Les PAIS répondent ainsi au souci manifesté de travailler directement avec les incréments $\Delta V$ qui entrent dans la définition des Leq, à leur décharge peut-être y a t'il leur relative complexité et une certaine méconnaissance. 
I.2 - Quoiqu'il en soit la forme générale des PAIS $X_{1}$ positifs et du second ordre est donnée par l'intermé-

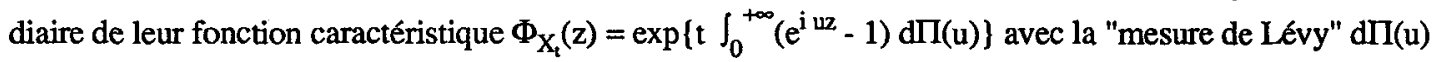
sur $R^{+}$telle que $U_{n}=\int_{0}^{+\infty} u^{n} d \Pi(u)$ est finie pour $n=0,1$ et 2 . On en déduit en particulier que les cumulants $\kappa_{n, \Delta X}$ de la loi de tout incrément $X_{h+t}-X_{h}$ sont égaux à $t U_{n}$, (si ils sont définis pour $n \geq 3$ ), et notamment l'espérance mathématique $E\left(X_{h+t}-X_{h}\right)=t U_{1}$ et la variance $\operatorname{var}\left(X_{h+t}-X_{h}\right)=t U_{2}$. Quand on passe à la loi des accroissements moyens nous avons donc $E(\Delta / V / \Delta t)$ noté $E_{V}=U_{1}, \operatorname{var}(\Delta / V / \Delta t)$ noté $\sigma_{V}{ }^{2}=U_{2} / \Delta t$ et accessoirement les cumulants $\kappa_{n V}$ égaux à $U_{n} / \Delta t^{n-1}$.

I.3 - Par ailleurs les niveaux mesurés Leq $=10 \log (\Delta \mathrm{V} / \Delta \mathrm{t})$ sont des variables transformées des accroissements moyens $\Delta f \mathrm{~V} / \Delta \mathrm{t}$ selon l'application $f()=.10 \log ()=.1 / c \log ($.$) avec c=1 / 10 \log 10$ \# 0,23. Les $\operatorname{Leq}$ successifs sont également indépendants et suivent une autre loi commune avec l'espérance $\mathrm{E}_{\mathrm{L}}$, la variance $\sigma_{\mathrm{L}}{ }^{2}$ et accessoirement les cumulants ultérieurs $\kappa_{\mathrm{qL}}$. Ce sont les mesures observées et c'est à partir de leur relevé que l'on peut estimer les paramètres $\mathrm{E}_{\mathrm{L}}, \sigma_{\mathrm{L}}{ }^{2} \ldots$; en dernier l'acousticien est davantage intéressé par la connaissance de $f\left(E_{V}\right)$ que par celle de $E_{L}$ puisque $f\left(E_{V}\right)=10 \log \{E(\Delta V / \Delta t)\}$ a la même définition qu'un Leq. Nous en déduisons en particulier les développements suivants (on rappelle que $\mathrm{c}<1$ ) :

$$
\begin{aligned}
& \mathrm{f}\left(\mathrm{E}_{\mathrm{V}}\right)=\mathrm{E}_{\mathrm{L}}+\sum_{\mathrm{q} \geqslant 2} \mathrm{c}^{\mathrm{q}-1} \kappa_{\mathrm{qL}} / \mathrm{q} ! \\
& \sigma_{\mathrm{V}}{ }^{2} / \mathrm{E}_{\mathrm{V}}{ }^{2}=\mathrm{c}^{2}\left\{\kappa_{2 \mathrm{~L}}+\mathrm{c} \kappa_{3 \mathrm{~L}}+\mathrm{c}^{2} / 12\left(7 \kappa_{4 \mathrm{~L}}+6 \kappa_{2 \mathrm{~L}}{ }^{2}\right)\right\}+\mathrm{O}\left(\mathrm{c}^{5}\right) \\
& \mu_{3 \mathrm{~V}} / \mathrm{E}_{\mathrm{V}}{ }^{3}=\kappa_{3 \mathrm{~V}} / \mathrm{E}_{\mathrm{V}}{ }^{3}=\mathrm{c}^{3}\left(\kappa_{3 \mathrm{~L}}+3 \mathrm{c} / 2\left(\kappa_{4 \mathrm{~L}}+2 \kappa_{2 \mathrm{~L}}{ }^{2}\right)\right\}+\mathrm{O}\left(\mathrm{c}^{5}\right), \text { etc } \ldots
\end{aligned}
$$

avec au premier membre les premiers moments centrés de la loi des accroissements moyens $\Delta \sqrt{ } \mathrm{V} / \Delta t$ et dans le second membre les cumulants $\kappa_{\mathrm{qL}}$ de la loi des niveaux mesurés $\mathrm{L}_{\mathrm{i}}$. Sur le plan Statistique les statistiques $\mathbf{k}_{\mathrm{qL}}$ de Fisher sont des estimateurs sans biais des cumulants $k_{\mathrm{qL}}$, et celles de Dwyer et Dressel $\mathbf{k}_{\mathrm{qprL}}$ pour les produits $\kappa_{\mathrm{qL}} \kappa_{\mathrm{pL}} \kappa_{\mathrm{rL}}$, etc ... ; on connaît également les variances de ces estimations et leurs propres estimations, ... (expressions connues, rappelées par exemple dans [7]).

\section{II - DES MESURES DE BRUIT ROUTIER.}

II.1 - Par conséquent les quantités $\mathrm{f}\left(\mathrm{E}_{\mathrm{V}}\right), \sigma_{\mathrm{V}}{ }^{2} / \mathrm{E}_{\mathrm{V}}{ }^{2}$, voire $\kappa_{3 \mathrm{~V}} / \mathrm{E}_{\mathrm{V}}{ }^{3}$ qui sont attachées à la loi des accroissements moyens $\Delta \mathrm{V} / \Delta \mathrm{t}$ sont simplement estimées à partir des niveaux mesurés $\mathrm{L}_{\mathbf{i}}$, avec les statistiques $\mathrm{k}_{\mathrm{qL}}$ de Fisher et $\mathbf{k}_{\mathrm{qprL}}$ de Dwyer et Dressel $\mathbf{k}_{\mathrm{qprL}}$ :

$\widehat{f\left(E_{\mathrm{V}}\right)}=\mathrm{E}_{\mathrm{L}}+\mathrm{c} \mathrm{k}_{2 \mathrm{~L}} / 2+\mathrm{c}^{2} \mathrm{k}_{3 \mathrm{~L}} / 6+\mathrm{c}^{3} \mathrm{k}_{4 \mathrm{~L}} / 24$

$\widehat{\sigma_{v}^{2} / E_{v}^{2}}=c^{2}\left\{k_{2 L}+c k_{3 L}+c^{2} / 12\left(7 k_{4 L}+6 k_{22 L}\right)\right\} \quad$ voire $\widehat{\kappa_{3 v} / E_{v}^{3}}=c^{3}\left\{k_{3 L}+3 c / 2\left(k_{4 L}+2 k_{22 L}\right)\right\}$

Les performances des sonomètres intégrateurs interviennent ici, puisque lorsque les niveaux sont enregis-

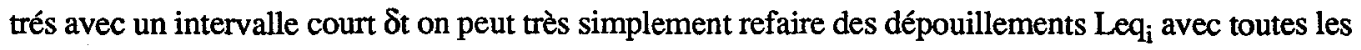
durées $\Delta t$ égales à des multiples $m \delta t\left(^{*}\right)$. D'après les propriétés rappelées ci-dessus $\widehat{f\left(E_{\mathrm{v}}\right)}, c_{2}=\Delta t \widehat{\sigma_{v}^{2} / E_{v}^{2}}$ et $c_{3}=\Delta t^{2} \widehat{\kappa_{3 v} / E_{v}^{3}}$ ne dépendent pas de $\Delta t$ (hormis les fluctuations d'échantillonnage qui deviennent d'autant

\footnotetext{
* Encore faut-il pouvoir relire les niveaux Leq calculés sur les différentes durées $\mathrm{m} \delta \mathrm{t}$; les sonomètres de nouvelle génération numérisent et stockent bien les niveaux, mais les mettent beaucoup plus difficilement à disposition pour d'autres logiciels sous forme de fichier en caractères Ascii (ce qui est pourtant indispensable pour bien des études !).
} 
plus importantes que $\Delta t$ augmente et donc que le nombre de niveaux $L e q_{i}$ diminue). Les variations en fonction de $\Delta t$ de ces estimations constituent la première vérification nécessaire d'un modèle de PAIS appliqué à lintégrale de la puissance acoustique $\int \mathrm{V}_{\mathrm{t}} \mathrm{dt}=\int\left(\mathrm{p}(\mathrm{t}) / \mathrm{p}_{0}\right)^{2} \mathrm{dt}$.

II.2 - Le bruit de la circulation se prête tout à fait à ces considérations. Nous avons réalisé des enregistements dans plusieurs sites routiers sur des périodes de plus d'une heure en moyenne (ou davantage) avec des sonomètres intégrateurs fonctionnant sur des périodes élémentaires $\delta \mathrm{t}$ de l'ordre de la demi-seconde ou moins. Nous avons ensuite calculé les estimateurs ci dessus en fonction de plusieurs durées multiples $\Delta \mathrm{t}=$ $\mathrm{m} \delta$ t et examiné leurs variations [8].

Les sites étaient contrastés. Avec des trafics faibles en champ réverbéré et diffus par exemple (rues étroites), chaque véhicule reste longtemps la source dominante (soit en champ direct, soit par l'intermédiaire de ses réflexions), et donc l'indépendance des diverses contributions $\Delta \int \mathrm{V}$ sur les $\Delta t$ successifs n'est guère raisonnable, on peut supposer que l'adéquation à un modèle de PAIS pour l'intégrale $\int V_{t} d t$ n'est pas satisfaisante. A l'inverse avec un trafic important et en champ libre, les véhicules ne peuvent être dominants sur une longue durée et sont rapidement masqués par de nouveaux véhicules à l'émission indépendante, l'absence de réverbération ne permet pas non plus à l'émission de chaque véhicule de revenir contribuer de manière importante dans le $\Delta V \mathrm{~V}$ de nombreux intervalles $\Delta \mathrm{t}_{\mathrm{i}}$; en conséquence l'hypothèse que les $\Delta / \mathrm{V}$ sont indépendants et suivent une même loi (trafic régulier pendant les heures d'activité) est beaucoup plus crédible.

Les résultats sont illustrés dans les figures ci-contre :
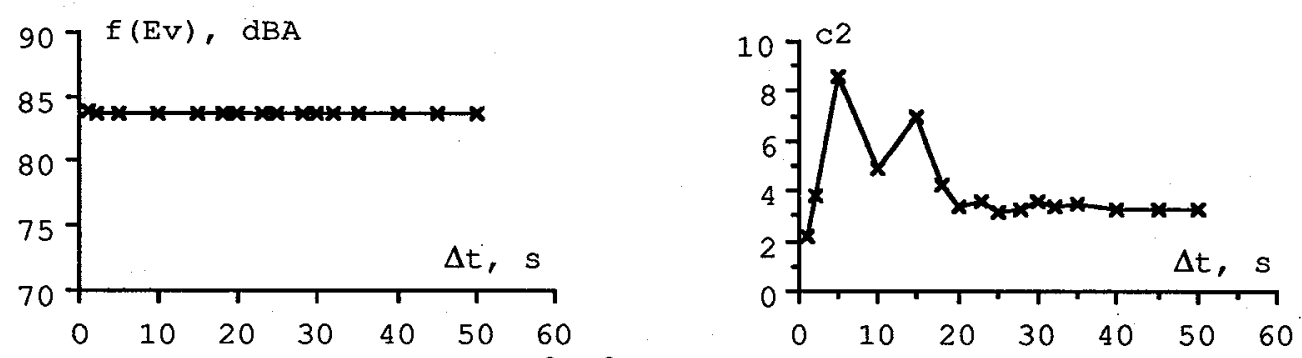

Figure 1 - Estimations de $f\left(\mathrm{E}_{\mathrm{V}}\right)$ et $c_{2}=\Delta t \sigma_{\mathrm{V}}{ }^{2} / \mathrm{E}_{\mathrm{V}}^{2}$ pour des niveaux enregistrés à proximité d'un axe autoroutier chargé, en champ libre ; $f\left(E_{V}\right)$ and $c_{2}=\Delta t{\sigma_{V}}^{2} / E_{V}^{2}$ estimations near by highway.
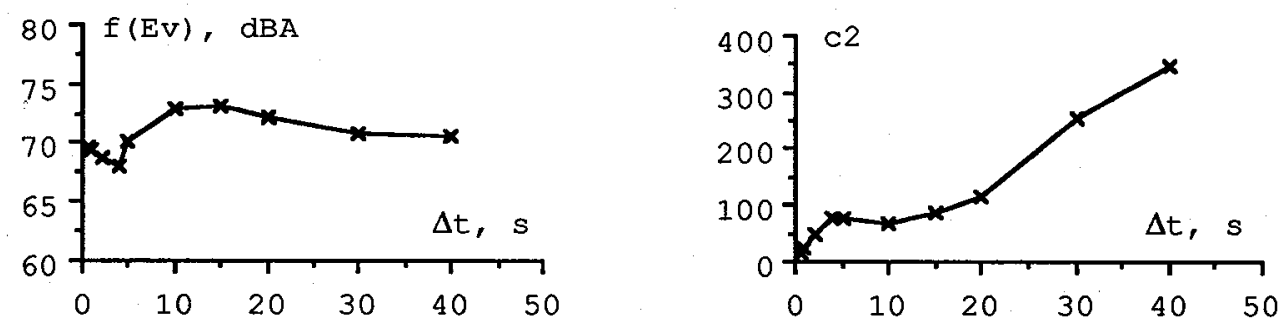

Figure 2 - Niveaux dans une rue en $U$ avec un débit moyen ; estimations in a "canyon" street.

Ce sont deux résultats types, le trafic dense en champ libre montre une estimation $\widehat{\mathrm{f}\left(\mathrm{E}_{\mathrm{v}}\right)}$ constante et une estimation $\Delta \mathrm{t} \widehat{\sigma_{\mathrm{v}}^{2} / \mathrm{E}_{\mathrm{v}}^{2}}$ constante à partir d'une durée $\Delta \mathrm{t}$ de $20 \mathrm{~s}$. Le trafic moyen avec réflexions montre une 
adéquation au modèle différente, $\widehat{\mathrm{f}\left(\mathrm{E}_{\mathrm{v}}\right)}$ varie en fonction de $\Delta t$ et $\Delta t \widehat{\sigma_{\mathrm{v}}^{2} / \mathrm{E}_{\mathrm{v}}^{2}}$ est croissante ; pour leur part les estimations $\Delta t^{2} \widehat{\kappa_{3 v} / E_{v}^{3}}$ sont un peu plus dispersées mais se comportent comme les précédentes, la première est sensiblement constante en champ libre pour des $\Delta t$ supérieurs à $20 \mathrm{~s}$, la seconde est franchement croissante dans la rue en $U$.

\section{CONCLUSION.}

Ces exemples types résument l'ensemble des mesures. Les sites qui se prêtent le mieux à l'indépendance montrent une assez bonne adéquation avec un PAIS pour l'intégrale $\int \mathrm{V}_{\mathrm{t}} \mathrm{dt}$, les $\left.\widehat{\left(\mathrm{E}_{\mathrm{v}}\right.}\right)$ sont quasiment constants et les $\Delta t \widehat{\sigma_{\mathrm{v}}^{2} / \mathrm{E}_{\mathrm{v}}^{2}}$ le sont de manière satisfaisante à partir d'une durée minimale qui est de l'ordre de $10 \mathrm{a}$ 20 s. Cela signifie que les puissances acoustiques ne suivent pas véritablement un modèle aussi strict (l'indépendance des incréments de $\int V_{t} d t$ sur tous intervalles disjoints), mais que cependant pour un grand nombre de trafics routiers le modèle se révèle bien adapté, notamment il se prête bien aux trafics routiers moyens et importants dès que la durée d'intégration $\Delta \mathrm{t}$ est de l'ordre de $10 \mathrm{à} 20 \mathrm{~s}$, et quelques fois aussi à d'autres situations.

Les PAIS sont une possibilité nouvelle (?) pour modéliser les niveaux de bruit quand on les applique au processus intégral $\int V_{t} \mathrm{dt}$. Ce modèle est tout à fait en accord avec les possibilités des sonomètres actuels, et il a déja permis de discuter une soi-disant hypothèse à propos du caractère gaussien des niveaux de bruit routiers $[7,9,10]$. On peut remarquer en dernier que les modèles habituels s'appliquent directement aux rapports $\Delta\left(\int \mathrm{V}_{\mathrm{t}} \mathrm{dt}\right) / \Delta \mathrm{t}=\Delta\left(\int\left(\mathrm{p}_{\mathrm{t}} / \mathrm{p}_{0}\right)^{2} \mathrm{dt}\right) / \Delta \mathrm{t}$ et conduisent de ce fait à la pression efficace $\overline{\mathrm{p}^{2}} / \mathrm{p}_{0}^{2}$ sur la durée $\Delta \mathrm{t}$, laquelle n'est évidemment pas additive dans le temps ; à l'inverse les PAIS pour $\Delta\left(j V_{t} d t\right)$ mettent l'accent sur le seul numérateur, c'est ce qui permet de développer des propriétés liées à la seule additivité de variables aléatoires indépendantes, le passage aux accroissements moyens (division par $\Delta t$ ) n'intervient qu'en dernier après que l'on a pu mettre à profit les propriétés des PAIS.

\section{REFERENCES BIBLIOGRAPHIQUES.}

[1] Maurin M., 1991, Enquête et campagne de mesures sur le bruit de circulation en France, Recherche Transports et Sécurité $\mathrm{n}^{\circ} 32$.

[2] Josse R., 1973, Notions d'acoustique à l'usage des architectes Ingénieurs et urbanistes, Eyrolles.

[3] Lamure C., 1975, Noise emitted by road traffic, dans Alexandre, Barde, Lamure, Langdon Road traffic noise, chap. 5, Applied Science Publishers.

[4] Migneron J.G., Acoustique urbaine, 1980, Masson, Presses de l'Université Laval - Québec.

[5] Bastide J.C., 1988, Estimation et mesure du niveau acoustique continu équivalent, Revue de Statistique Appliquée, vol XXXVI n ${ }^{\circ}$, pages 5-14.

[6] Bouleau N, 1988, Processus stochastiques et Applications, Hermann.

[7] Maurin M., 1990, L'échantillonnage des niveaux de bruit et la normalité, Journées d'étude Métrologie et Exposition Sonore, INRS, Nancy 1990.

[8] Messiaen L., 1991, Analyse statistique des bruits de la circulation automobile, Rapport de stage ECL, INRETS.

[9] Maurin M., 1990, A propos d'une hypothèse gaussienne en Acoustique, 1er Congrès de la SFA, Lyon 1990.

[10] Maurin M., 1991, Noise levels distributions in Environmental Acoustic, Western Pacific Regional Acoustics Conference IV, Brisbane, nov. 1991. 